\title{
Pensando de Manera Crítica sobre el Pensamiento Crítico
}

\author{
E. Wayne Ross $\left(^{*}\right)$ y Michelle Gautreaux $\left(^{*}\right)$ \\ $\left.{ }^{*}\right)$ University of British Columbia - Vancouver - Canadá
}

\section{RESUMEN}

Desde hace tiempo la manera dominante para enseñar el pensamiento crítico en las escuelas ha sido a través de un enfoque basado en el desarrollo de habilidades. Este enfoque pone su énfasis en la instrucción directa para enseñar habilidades de pensamiento crítico discretas, ofreciendo a los alumnos oportunidades para practicar la aplicación de habilidades de pensamiento y ayudándoles a trasladar habilidades de pensamiento crítico de un contexto a otro. En este trabajo, se propone construir el pensamiento crítico desde un enfoque más holístico basado en la dialéctica, que complemente, o más bien sustituya, la lógica formal con un enfoque que analice la forma en que están las cosas, sus historias y la manera en que las cosas cambian.

Palabras Clave: Pensamiento crítico, Enfoque dialéctico.

\section{Thinking Critically about Critical Thinking}

\section{ABSTRACT}

For a long time the dominant way to teach critical thinking in schools has been a way with a focus on skill development. This approach emphasizes direct instruction to teach discrete critical thinking skills, offering students opportunities to practice applying thinking skills and helping them transfer critical thinking skills from one context to another. In this paper, we propose to conceive critical thinking with a more holistic approach based on dialectics, which complements, or rather substitutes, formal logic with an approach that analyzes the way things are, their histories and the way in which things change in the world.

Keywords: Critical thinking, Dialectical approach.

El pensamiento crítico generalmente se considera un elemento esencial de la 'competencia cívica'-: la capacidad de la gente para enfrentar problemas sociales persistentes y complejos. A pesar de un énfasis retórico en el pensamiento crítico, los investigadores han encontrado muy poca evidencia de la enseñanza del pensamiento crítico en las aulas de los colegios, incluso en las disciplinas académicas dirigidas hacia la preparación de los alumnos para la participación en la vida cívica.

El estilo pedagógico dominante en las aulas de los colegios sigue estando caracterizado por un estilo tradicional. El docente dirige toda actividad en la clase, normalmente situado de pie en frente de los alumnos sentados en filas de mesas. El temario deriva del libro de texto y se mide el aprendizaje de los alumnos por su capacidad para memorizar información basada en hechos discretos y desconectados. Aunque ha recibido muchas críticaseste estilo pedagógico y varias alternativas han sido presentadas, este estilo didáctico persiste en la impartición de las asignaturas, incluso en las ciencias sociales (Stanley, 1991).

Se puede explicar esta discrepancia entre la retórica y la realidad del pensamiento crítico en las aulas, por parte, tomado en cuenta varios factores, que incluyen: las influencias poderosas que tienen la organización, cultura, y arquitectura de los colegios y las universidades; las ideas aceptadas sobre la función de las universidades en la sociedad, y el énfasis que se pone en las notas que sacan los alumnos en los exámenes en vez de representaciones más auténticas de su aprendizaje de la materia. Sin embargo, el obstáculo más importante al que nos enfrentamos en nuestros esfuerzos para poner en práctica el pensamiento crítico es nuestra concepción de lo que es 'el pensamiento crítico'. En lo que sigue, resumiremos brevemente las deficiencias en nuestra concepción contemporánea del pensamiento crítico y propondremos una alternativa radical.

\section{El problema del pensamiento "no dialéctico"}

Buscando una definición de qué consiste el pensamiento crítico, muchos educadores acuden a la obra del filósofo americano John Dewey. En su obra Cómo Pensamos (How We Think es el título original en inglés, 1933), Dewey describe el pensamiento crítico (él siempre lo llamaba 'pensamiento reflexivo') como: 
El examen activo, persistente, y cuidadoso de toda creencia o supuesta forma de conocimiento a la luz de los fundamentos que la sostienen y las conclusiones a las que tiende ... (p. 8).

Hay dos obstáculos principales en la realización de este tipo de pensamiento en las aulas de las escuelas. Primero, la forma holística que tenía Dewey de concebir el pensamiento- lo cual no separa el conocer del hacer- tiende de ser tratada como una serie de pasos mecánicos para que los alumnos los sigan. Dewey presenta los elementos de pensamiento crítico como:

(1) sugerencias, en las que la mente salta hacia adelante en busca de una solución posible; (2) una intelectualización de la dificultad o complejidad que se ha experimentado (es decir, vivido directamente) en un problema que hay que resolver, una pregunta a la que hay que buscar la respuesta; (3) el uso de una sugerencia tras otra como hipótesis para iniciar y guiar la observación y otras operaciones en la recogida de material objetivo; (4) la elaboración mental de la idea a suposición como idea o suposición (razonamiento, en el sentido de que el razonamiento es una parte de la deducción y no su totalidad) y (5) la comprobación de la hipótesis mediante la acción real o imaginada (Dewey, 1933, p. 106).

Dewey nunca sugirió que el pensamiento crítico se resulte en pasos consecutivos mecánicos, o que el pensamiento crítico se resulte de la aplicación discreta de habilidades cognitivas para resolver un problema, pero muchas veces, profesores y educadores le interpretan a Dewey de esta manera. Con el paso de los años, muchos formadores de docentes en las universidades han alentado a los docentes a adoptar un enfoque basado en habilidades para enseñar el pensamiento crítico en las asignaturas de estudios sociales. La idea que subraya esta forma de enseñar se basa en la idea de que los profesores deben de usar la instrucción directa para enseñar habilidades discretas de pensamiento crítico, dándoles a sus alumnos muchas oportunidades para practicar la aplicación de habilidades de pensamiento y ayudándoles a transferir habilidades de pensamiento crítico de un contexto a otro. La fuente más importante e influyente sobre las concepciones tradicionales de la educación del pensamiento crítico es la Taxonomía de Objetivos Educativos (Taxonomy of Educational Objectives) de Benjamin Bloom (1956). Otros trabajos influyentes que promueven la enseñanza del pensamiento crítico como habilidades discretas son Desarrollando Programas de Destrezas de Pensamiento (Developing Thinking Skills) de Barry K. Beyer (1988) y Pensamiento Crítico (Critical Thinking) de Robert H. Ennis (1988).

Como resultado del enfoque tradicional del pensamiento crítico como el desarrollo de habilidades discretas, el ejercicio del juicio crítico muchas veces se reduce a criterios simples para evaluar pequeños trozos discretos de información. Por ejemplo, los profesores muchas veces ponen en práctica sistemas o estructuras que identifican las habilidades cognitivas o aspectos de pensamiento crítico que están vinculados a la noción de un argumento lógico:

- Comprender el significado de una declaración

- Distinguir entre hechos verificables y declaraciones de valores

- Distinguir las observaciones o razones relevantes de las irrelevantes

- Determinar la precisión fáctica de una declaración

- Determinar la credibilidad de una fuente de información

- Identificar declaraciones ambiguas
- Identificar una suposición tácita/ no declarada

- Detección de prejuicio o parcialidad

- Reconocer inconsistencias lógicas en una línea de razonamiento

- Juzgar si hay ambigüedad en una línea de razonamiento

- Juzgar si ciertas declaraciones se contradicen entre sí

- Juzgar si una conclusión sigue necesariamente/ como debería

- Juzgar si una declaración es lo suficientemente específica

- Juzgar si una declaración es realmente la aplicación de una cierta creencia

- Juzgar si una declaración de observación es confiable

- Juzgar si se justifica una conclusión inductiva.

- Juzgar si el problema ha sido identificado

- Juzgar si algo es una suposición.

- Juzgar si una definición es adecuada/suficiente.

- Juzgar si una declaración hecha por una supuesta autoridad es aceptable.

El problema con los paradigmas de pensamiento crítico que se enfocan en el desarrollo de habilidades genéricas que se pueden trasladar de un contexto a otro es que se deja al lado el elemento clave en el desarrollo del pensamiento crítico del alumnado, es decir, el conocimiento.

En una revisión de la investigación sobre el pensamiento crítico, Stanley (1991) concluye que "los intentos de enseñar habilidades de pensamiento genéricas o modelos sin una atención adecuada al contenido tienen pocas probabilidades de tener un impacto en el rendimiento del estudiante en las áreas temáticas" (p.255). Estudios académicos recientes indican que los alumnos más hábiles en el uso del pensamiento crítico para resolver problemas tenían tanto un conocimiento detallado de la materia relevante como una buena comprensión de las estrategias de resolución de problemas.

El problema fundamental con los enfoques tradicionales de enseñar el pensamiento crítico es el enfoque lineal, o 'no-dialectico'; es decir, la separación de lo que no se puede separar, con la consiguiente distorsión. Los alumnos deben tener la oportunidad de establecer conexiones entre el conocimiento previo y los diversos elementos del conocimiento nuevo, en lugar de aprender habilidades de forma aislada o examinar solo fragmentos desconectados de la información. Como ha comentado Bertell Ollman (1993; 2003), la mayoría de la gente puede ver las partes lo suficientemente bien, pero no las conexiones y los patrones generales de la existencia humana.

\section{El pensamiento Crítico como Pensamiento Dialéctico}

La insuficiencia del pensamiento no dialéctico, o el pensamiento desconectado, es particularmente evidente en las disciplinas donde se espera que los estudiantes se enfrenten problemas sociales persistentes y complejos. Si definimos la competencia cívica como la capacidad de comprender el mundo y tomar medidas para cambiarlo, es entonces imprescindible que comprendamos las diferencias entre obtener los hechos correctos, explicar los hechos y construir acciones prescriptivas.

Por ejemplo, muchas personas de diversas tendencias políticas han notado la paradoja de la creciente riqueza de unos pocos y la creciente pobreza de la mayoría de las personas, así como las conexiones entre los intereses de las empresas multinacionales y las acciones de los gobiernos o de ser pobres y sin poder. Como explica Ollman (1993), a pesar de la conciencia de estas relaciones sociales, la mayoría de la gente no se toma en serio tales observaciones. Al faltar una teoría para dar sentido a lo que está viendo, 
la gente no sabe la importancia que se le debe dar al asunto; se le olvida lo que acaba de ver o elimina las contradicciones, nombrándolas 'una paradoja'. El problema es que la socialización a la que nos sometemos (dentro y fuera del colegio), nos incita a enfocarnos en las particularidades de nuestras circunstancias y no hacerles caso a las conexiones entre las varias experiencias que tenemos. Por lo tanto, perdemos los patrones que surgen de las relaciones sociales. Los enfoques basados en el desarrollo de las habilidades para la educación del pensamiento crítico tienen un papel importante porque refuerzan esta tendencia. Además, las ciencias sociales dividen el conocimiento humano en varias disciplinas (historia, antropología, sociología, geografía, etc.), cada una con su propio lenguaje y formas de conocimiento específicos, lo que fomenta el foco en pequeños trozos y pedazos de experiencia humana. Lo que existía antes se toma como dado por hecho e inmutable. Por tanto, grandes cambios económicos y políticos a lo largo de la historia (como las revoluciones de 1789, 1848,1917, y 1989) son tratados como anomalías que requieren explicación.

En contraste, el pensamiento dialéctico implica un esfuerzo por comprender el mundo en términos de interconexiones- los vínculos entre las cosas tal como son ahora, sus propias precondiciones (es decir, de dónde vienen), y sus posibilidades en el futuro. El método dialéctico toma el cambio como punto de partida, y trata la estabilidad aparente como aquello que tiene que ser explicado (y la dialéctica facilita conceptos y marcos teóricos especializados para explicarlo). El pensamiento dialéctico es una orientación para comprender el mundo que requiere no solamente mostrar los hechos que están ocultos a la vista, sino también una comprensión más interconectada de los hechos que ya conocemos.

El problema es que la realidad es más que las apariencias. Enfocarse exclusivamente en las apariencias, es decir, como explica Ollman, centrarse solamente en la evidencia que nos llama la atención inmediata y directamente, puede ser engañosa. Basar nuestra comprensión de nosotros mismos y del mundo en solo lo que vemos, oímos, o tocamos en nuestro entorno inmediato, puede llevarnos a sacar conclusiones falsas o distorsionadas.

Comprender cualquier cosa en nuestra experiencia cotidiana requiere que sepamos algo sobre cómo surgió y se desarrolló y cómo se encaja en el contexto o sistema del que forma parte. Pero reconocer solo esto, sin embargo, no es suficiente ... Después de todo, pocos negarían que todo en el mundo está cambiando y que todo está relacionado de alguna forma u otra, y que la historia y las conexiones sistémicas le pertenecen al mundo real. La dificultad ha sido siempre el cómo pensar en las cosas de forma adecuada, cómo no distorsionarlas y cómo darles la atención y el peso que les merecen (Ollman, 1993, p. 11).

La dialéctica, explica Ollman, consiste en un intento de resolver esta dificultad, expandiendo nuestra comprensión de 'cualquier cosa' para incluir (como aspectos de lo que es) tanto el proceso por el cual se ha convertido en esa cosa como el contexto interactivo más amplio en el que se encuentra. La dialéctica reestructura el pensamiento sobre la realidad. Reemplaza la noción de sentido común de 'cosa' como algo que tiene una historia y tiene conexiones externas con otras cosas, con nociones de 'proceso' (que contiene su historia y posibles futuros) y 'relación' (que contiene como parte de lo que es sus vínculos con otras relaciones). Como dice Sciabarra, la dialéctica es el "arte de mantener el contexto", es decir, el arte de siempre tener en cuenta el contexto.
[El método dialéctico] nos aconseja estudiar el objeto de nuestra investigación desde el punto de vista de varias perspectivas y niveles de generalidad, a fin de llegar a tener una idea comprensiva y más completa de la misma. Este tipo de investigación requiere que entendamos el objeto de estudio en términos del sistema más grande en el que está situado, así como su desarrollo a lo largo de la historia. Debido a que los seres humanos no son omniscientes, porque ninguno de nosotros puede ver el "todo" como si fuéramos dioses desde una perspectiva divina "sinóptica", solo a través de la abstracción selectiva podemos construir una comprensión más integrada y completa del fenómeno que estamos investigando, una comprensión de sus condiciones antecedentes, sus relaciones con otros tipos de fenómeno y sus tendencias (2005, p. 8).

La abstracción, que forma parte integral del método dialectico, puede ser explicada con una analogía; es como usar los lentes de una cámara con diferentes distancias focales: el lente 'zoom' para enfocar un objeto distante (preguntamos, ¿cuál es la historia de esta cosa que estoy observando?), o usar un lente panorámico para poder captar más bien la escena o el contexto (¿cuál es el contexto social del problema ahora?). Esto nos plantea preguntas importantes, como: ¿Por dónde comenzar? y ¿Qué debería buscar?

A diferencia del pensamiento no dialéctico, donde uno comienza con una pequeña parte y mediante el establecimiento de conexiones trata de reconstruir el todo más grande, el pensamiento dialéctico comienza con el todo (o lo que uno entiende del todo) y luego examina la parte para ver dónde se encaja, y cómo funciona, lo que finalmente lleva a una comprensión más completa del todo. El ejemplo por excelencia de este modo de pensar es la obra de Karl Marx; para Marx, el capitalismo fue el punto de comienzo para una investigación de cualquier cosa que tenga lugar dentro del él. (Aunque es importante notar que la mayor parte de la teoría de la dialéctica de Marx la tomó de Georg Wilhelm Friedrich Hegel, quien sistematizó una forma de pensar que tiene su origen en el pensamiento de los antiguos griegos). Además, filósofos no-marxistas como Alfred North Whitehead y F. H. Bradely desarrollaron sus propias versiones del método dialectico.

Las investigaciones dialécticas proceden empezando con el todo hacia la parte (es decir, desde el sistema entero hacia dentro) y están principalmente dirigidas al análisis de cuatro tipos de relaciones. La primera relación trata de identidad y diferencia; es decir, cómo las cosas son iguales/idénticas o diferentes, pero no ambas. Por ejemplo, hay diferencias entre ganancias, renta e interés; sin embargo, un análisis dialéctico demuestra la identidad de cada concepto como formas de plusvalía, es decir, riqueza creada y producida por los trabajadores que al final no se les devuelve en forma de salarios.

La segunda relación es la interpretación de opuestos, es decir, cómo se nos aparece una cosa y cómo funciona se puede atribuir a las condiciones que la rodean. Por ejemplo, un capitalista ve una máquina como una mercancía, comprada en el mercado y como algo que le va a brindar un beneficio. Mientras, el trabajador mira la misma máquina, pero ve un instrumento que va a determinar sus movimientos en el proceso de producción.

La tercera relación es cantidad-calidad. La cantidad se convierte en calidad y las cualidades se convierten en cantidades. La fuerza motriz del cambio es la incorporación de cantidades específicas que causan un cambio. Por ejemplo, reducir la temperatura del agua produce una calidad nueva, el hielo. Añadir años a la vida crea una calidad nueva. Añadir sal a la comida, cerca- 
nía a la amistad, velocidad a una bala, instrumentos a un grupo musical, todo ello hace algo nuevo. El cambio gradual, entonces, está acompañado por cambio cualitativo -revolucionario- lo que parece como un salto aparentemente inesperado.

Por último, y lo más importante, es la contradicción. La contradicción es el desarrollo incompatible de elementos diferentes dentro de la misma relación (la unidad y lucha de los opuestos). Todas las cosas consisten en contradicciones. Algunos ejemplos simples de polaridades unificadas incluyen:

- La anatomía: el pulgar y el dedo índice

- Las matemáticas: sumar/ restar; multiplicar/ dividir

- La educación: la naturaleza y la crianza

- La música: clave principal/ clave menor; el sonido/ el silencio

- La literatura: los mejores de los tiempos; los peores de los tiempos

- La física: toda acción tiene una reacción

Ollman explica que "los pensadores no-dialécticos en cada área de la investigación intelectual siempre están buscando el 'agitador que viene de fuera'; buscan algo o alguien que viene de fuera del problema que se examina y que es la causa de lo que esté ocurriendo. En cambio, los pensadores dialécticos les atribuyen la mayoría de la responsabilidad de todo tipo de cambio a las contradicciones internas del sistema (o sistemas) en los que ocurre" (1993, p. 16).

Sin una concepción de las cosas como relaciones, es difícil enfocarse en los lados diferentes de una contradicción a la vez. Incluso si se examinan todos los lados de una contradicción, no se les puede dar a cada uno el mismo nivel de atención. Por tanto, muchas veces su interacción mutua se confunde con causalidad. Para los intelectuales no-dialécticos, contradicciones reales -como, por ejemplo, el hecho de que durante el 'boom económico' de los años 80 y los 90, cuando el Producto Interno Bruto de los Estados Unidos se aumentó 25 por ciento, la tasa de pobreza entre los trabajadores aumentó 7,4 por ciento, o que mientras los ricos se han hecho bastante más ricos, cuatro de cada cinco hogares en los EEUU se llevan a casa una ración más pequeña del 'pastel económico' desde 1977- solo se pueden entender como diferencias, paradojas, oposición, desequilibrios; mientras las fuerzas subyacentes responsables de estas apariencias se mantienen invisibles o no reconocidos.
El pensamiento dialéctico no se trata de una cuestión simple $\mathrm{y}$, como el pensamiento no dialéctico, existen varias distorsiones asociadas con este modo de pensar. Si los intelectuales no-dialécticos tienden a enfocarse en los detalles y no son capaces de ver el todo de un problema, los pensadores dialécticos muchas veces hacen lo contrario, no haciéndoles caso a los detalles en favor de las generalizaciones.

El método dialéctico, sin embargo, es una manera de entender el ámbito entero de los cambios e interacciones que ocurren en el mundo. Si queremos que nuestros alumnos sean capaces de entender el mundo y tomar medidas para cambiarlo, la dialéctica nos ayuda a plantear preguntas que hacen posible la acción efectiva: ¿Qué tipo de cambios están ocurriendo ya? ¿Qué tipo de cambios son posibles? Lo único que no se puede escoger es lo que ya tenemos. El método dialectico es a la vez crítico y radical. Nos ayuda a entender el presente como un momento por el cual estamos pasando. La dialéctica nos obliga a examinar de dónde hemos venido y hacia dónde estamos yendo, como parte de nuestro aprendizaje sobre este mundo en que vivimos. Nos permite comprender que todo en este mundo -cada ser humano y cada cosa que existe- estamos conectados, unos con otros y que, sobre todo, somos capaces de cambiar nuestro mundo.

\section{Bibliografía}

Bloom, B. S. (1964). Taxonomy of educational objectives. New York, NY: Longmans, Green.

Beyer, B. K. (1988). Developing a thinking skills program. Boston, MA: Allyn and Bacon.

Dewey, J. (1933). How we think. Lexington, MA: Heath.

Ennis, R. H. (1995). Critical thinking. London: Pearson.

Ollman, B. (1993). Dialectical investigations. New York: Routledge.

Ollman, B. (2001). How to take and exam and remake the world. Montreal, QC: Black Rose.

Ollman, B. (2003). Dance of the dialectic: Steps in Marx's method. Urbana, Ill: University of Illinois Press.

Sciabarra, C. M. (2005, September 1). Dialectics and liberty: A defense of dialectical method in the service of a libertarian social theory. The Freeman. Retrieved from https://fee.org/articles/dialectics-and-liberty/

Stanley, W B. (1991). Teacher competence in social studies. In J. P. Shaver (Ed.), Handbook of social studies teaching and learning (pp. 249- 262). New York: Macmillan. 